\title{
Dynamic Collapse Analysis of Reticulated Shell Structures with Substructures
}

\author{
Li Hong-mei, Wang Jun-lin, Ren Xiao-qiang, \\ Sun Jian-heng \\ College of Urban and Rural Construction, \\ Agricultural University of Hebei, \\ Baoding 071001, China \\ xqren@126.com
}

\author{
Lu Wei \\ Engineering and Technical college of Hebei, \\ Cangzhou 061001, \\ China
}

\begin{abstract}
Dynamic collapse analysis is an important research subject for large span single layer reticulated shell structures. In this paper, the dynamic collapse behavior of the single layer reticulated shell with substructure which supports the reticulated shell is investigated under the earthquake actions. In the analysis, the geometric imperfections, the material and the geometric nonlinear of the structures are considered. The effects of the different stiffness of substructure to the collapse earthquake accelerations and the plastic member distribution of the reticulated shell are investigated.
\end{abstract}

Keywords - Single layer spherical reticulated shell; dynamic collapse; Substructures;plasticity ratio

\section{INTRODUCTION}

Reticulated shell structure is a basic type of the large spatial structures. And it is widely used in engineering due to its attractive architectural performance and the good load bearing capacity. Because the membrane force is the main resistance force of the reticulated shell structures under loads, the stability behavior of this type structure is a controlling factor in the analysis and design. The stability behavior includes static stability and dynamic stability. In the past decades, the static stability of the reticulated shell structures has been extensively studied, and a lot of research results have been got [1-4]. In recent years, the dynamic collapse of the reticulated shell caused by the earthquake action also attracts a lot of researchers, and a series of the investigation results have been presented [5-8]. But up to now, most of the dynamic collapse analysis papers considered only the reticulated shell itself and neglected the supporting frame structures, namely substructures.

In practical, most spatial structures have a supporting frame or called, "substructure". During an earthquake, the effects of seismic ground motions act on the base of these substructures and then these effects are transmitted up into the main reticulated shell structure. In this respect, an accurate and realistic investigation of the behavior of earthquake resistant spatial structures would be achieved if the reticulated shell structure and the supporting frame (substructure) are considered as an integral whole. To date, there are only a few papers published concerning this issue [9-11].
This paper considers the reticulated spherical shell structure and the substructures as an integral whole and investigates the dynamic collapse behavior of the reticulated shell under earthquake actions. In the analysis, the initial geometric imperfections together with geometric and material nonlinearities are all included, and the reticulated spherical shells with substructures of the different stiffness are analyzed to demonstrate the effects of stiffness on the dynamic collapse of the structures.

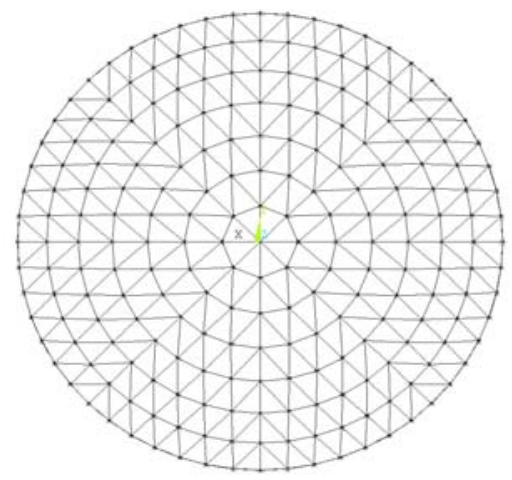

Fig. 1. K8 reticulated shell

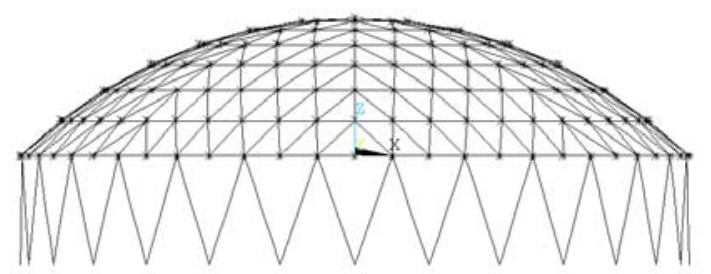

Fig. 2. K8 reticulated shell with substructure

\section{RETICULATED SHELl MODELS AND COLLAPSE ANALYSIS METHOD}

The widely used K8 reticulated spherical shell, as shown in Fig. 1 and Fig. 2, is used as the model structure in the numerical analysis. The model reticulated shell has a span of $50 \mathrm{~m}$ and rise of $10 \mathrm{~m}$ which give the structure a rise to span ratio of 0.2. The steel frame shown in Fig. 2 is used as the substructure to support the main reticulated spherical shell 
structure. The main reticulated spherical shell is rigid-jointed with the substructure. The substructure has a height of $8 \mathrm{~m}$ and is also rigid-jointed with the base.

A uniformly distributed load of $1.3 \mathrm{kN} / \mathrm{m}^{2}$ was assumed to be applied over the dome. The steel material used for the members of both the dome and substructure was Q235 with a modulus of elasticity $\mathrm{E}=206 \mathrm{MPa}$, Poisson ratio $v=0.26$, yield strength $\mathrm{f}_{\mathrm{y}}=235 \mathrm{MPa}$ and the material density is $7850 \mathrm{~kg} / \mathrm{m}^{3}$. All of the material was assumed to be perfectly elastic-plastic in behavior. The Rayleigh damping is used in the numerical analysis and a damping ratio of 0.02 was assumed.

Three type of tubular cross -sections are applied for the members of the reticulated spherical shell, and they are $\Phi 108 \times 4, \Phi 83 \times 4$ and $\Phi 70 \times 4$ respectively according to the internal force of members arising from static analysis. The ring beam of the substructure is made of steel with a 'I' section $250 \times 250 \times 10 \quad$ (flange) $\times 8 \quad($ web) cross-section. The cross sections of the members of the structure are also tubular cross sections and their dimension is given in the following section.

The numerical analysis of the structures is carried out by using the finite element analysis software ANSYS [12]. In the analysis by ANSYS, the PIPE20 element is used for all the tubular members. This element type can deal with both the geometric and material nonlinear behavior of the structure. The members of the main dome and the substructure are all rigidly connected. To model the weight of the structure for the seismic analysis, three-dimensional MASS21 elements are used to concentrate the weight of the structure onto the corresponding nodes.

The three dimensional El-Centro earthquake acceleration time series is selected as the input acceleration, in which the three peak accelerations of the time series in both horizontal and vertical directions are $\mathrm{a}_{\mathrm{x}}=2.1014 \mathrm{~m} / \mathrm{s}^{2}, \mathrm{a}_{\mathrm{y}}=3.4170 \mathrm{~m} / \mathrm{s}^{2}, \mathrm{a}_{\mathrm{z}}$ $=-2.0635 \mathrm{~m} / \mathrm{s}^{2}$, respectively [13]. Ten second time history duration is used so that all the peak accelerations are included in the analysis. For the main dome structure, a value of D/300 for the initial geometric imperfection was considered, and the first buckling mode is employed for the distribution of the imperfection.

In the numerical analysis, the Budinsky-Roth [14] criterion is used to determine the dynamic collapse acceleration of the main reticulated shell structure. By using this criterion, the seismic acceleration increases gradually by the same factor in three directions while the cycle of the time series is kept unchanged. The dynamic response of the structure is monitored under increasing acceleration, and a sudden increase of displacement due to a very small increase in the magnitude of the acceleration is considered as an indication of the dynamic collapse of the structure.

\section{DyNAMIC COLLAPSE ANALYSIS OF THE RETICULATED SHELL WITH SUBSTRUCTURE}

To demonstrate the effect of the substructure to the dynamic collapse of the main structure, the reticulated spherical shell without substructure is analyzed firstly. In the analysis, the reticulated spherical shell is pin connected with the base, and all the three translational displacements of the boundary nodes of the reticulated structures are restrained.

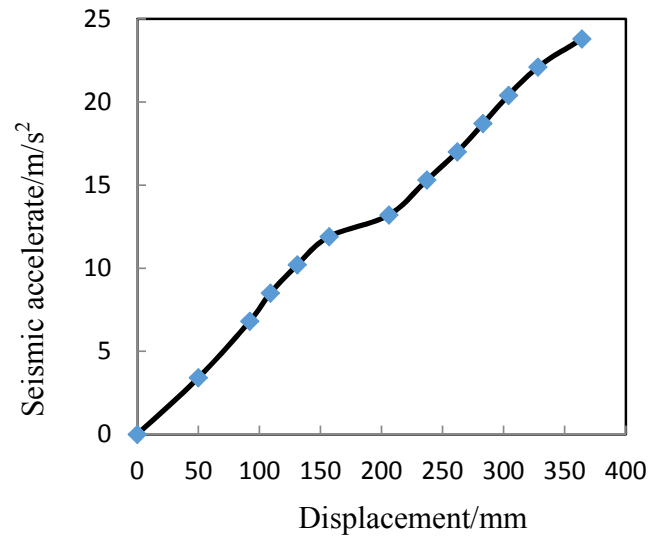

Fig. 3. Maximum displacement of the reticulated shell without substructure

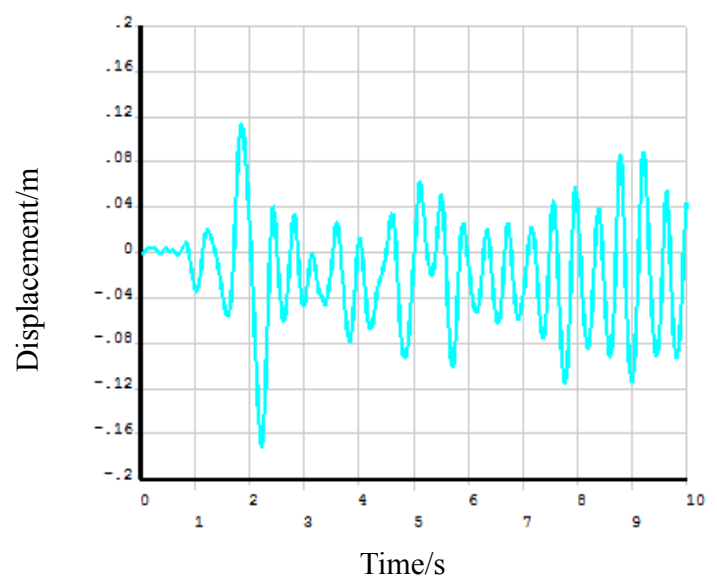

Fig. 4. Dynamic response of the maximum displacement of node 91

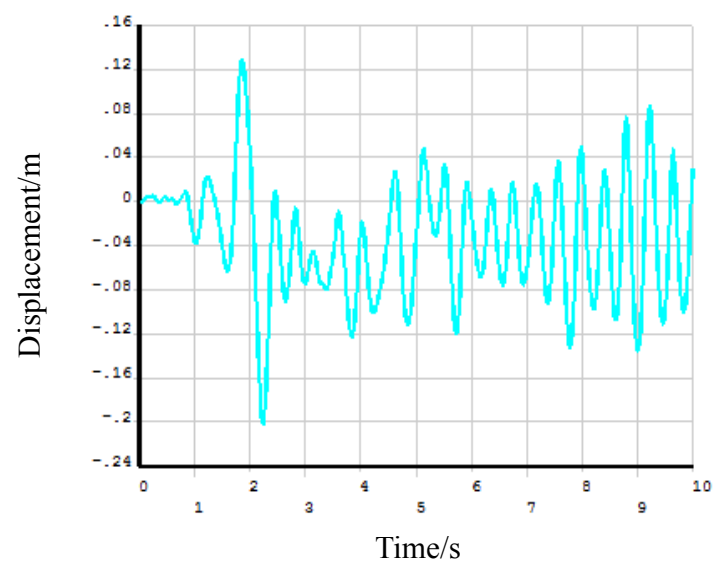

Fig. 5. Dynamic response of the maximum displacement of node 91

The numerical dynamic analysis results of the reticulated spherical shell without considering the substructure are shown in Fig. 3, Fig. 4 and Fig. 5. The result also shows that the maximum displacement occurs in the vertical displacement of node 91. Fig. 3 shows the variation of the maximum node displacement of the reticulated shell with the earthquake peak acceleration. The figure indicates that when the earthquake 
peak acceleration increases from $3.4 \mathrm{~m} / \mathrm{s}^{2}$ to $11.9 \mathrm{~m} / \mathrm{s}^{2}$, the maximum displacement increases from $50 \mathrm{~mm}$ to $157 \mathrm{~mm}$. The displacement increases nearly linearly with earthquake peak acceleration. When the earthquake peak acceleration increases from $11.9 \mathrm{~m} / \mathrm{s}^{2}$ to $13.2 \mathrm{~m} / \mathrm{s}^{2}$, the maximum displacement increases to $206 \mathrm{~mm}$ from $157 \mathrm{~mm}$, which is much larger than the increasing ratio of the earthquake acceleration. Fig. 4 shows that when the earthquake acceleration is $11.9 \mathrm{~m} / \mathrm{s}^{2}$, the dynamic response of the maximum displacement maintains the character of vibrating at its initial vibration equilibrium position. Fig. 5 shows that when the earthquake acceleration reaches $13.2 \mathrm{~m} / \mathrm{s}^{2}$, the dynamic response of the maximum displacement deviates from its initial vibration equilibrium position. Base on the Budinsky-Roth criterion, the collapse acceleration of the structure is between $11.9 \mathrm{~m} / \mathrm{s}^{2}$ and $13.2 \mathrm{~m} / \mathrm{s}^{2}$, and the average number $12.6 \mathrm{~m} / \mathrm{s}^{2}$ is taken as the dynamic collapse acceleration of the reticulated spherical shell without a substructure.

When the substructure is considered, the steel frame shown in Fig. 2 is used as the substructure. The tubular cross section of $\Phi 194 \times 8$ is adopted for all the columns of the substructure. The numerical analysis results are shown in Fig.6 and Fig.7. The maximum displacement under the action of earthquake occurs in the vertical displacement of node 53 instead of node 91 when the substructure is not considered.

Fig. 6 shows the maximum displacement of node 53 under different peak acceleration. When the peak acceleration increases from $3.4 \mathrm{~m} / \mathrm{s}^{2}$ to $9.2 \mathrm{~m} / \mathrm{s}^{2}$, the maximum displacement increase from $67 \mathrm{~mm}$ to $129 \mathrm{~mm}$, and when the peak acceleration increases from $9.2 \mathrm{~m} / \mathrm{s}^{2}$ to $9.5 \mathrm{~m} / \mathrm{s}^{2}$ only, the maximum displacement increases to $144 \mathrm{~mm}$ rapidly. Fig. 7 shows that the dynamic response of node 53 has seriously deviates from its initial vibration equilibrium position when the peak acceleration reaches $9.5 \mathrm{~m} / \mathrm{s}^{2}$. Based on the BudinskyRoth criterion, the dynamic collapse acceleration of the reticulated spherical shell with substructure of the cross section $\Phi 194 \times 8$ is $9.2 \mathrm{~m} / \mathrm{s}^{2}$, which is less $24.6 \%$ than the collapse acceleration without substructure.

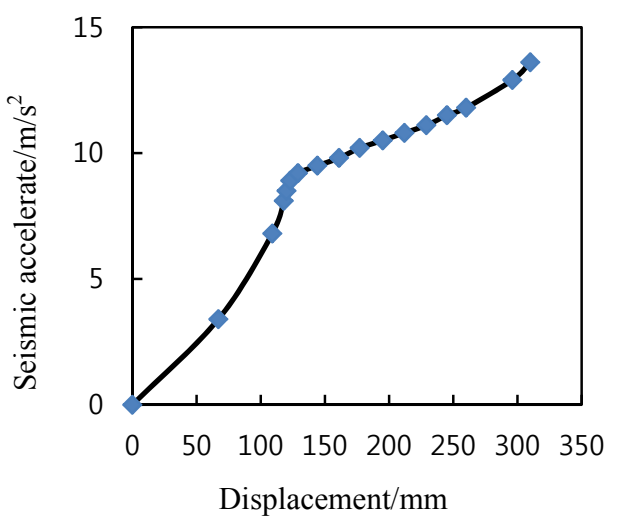

Fig. 6. Maximum displacement of the reticulated shell with substructure

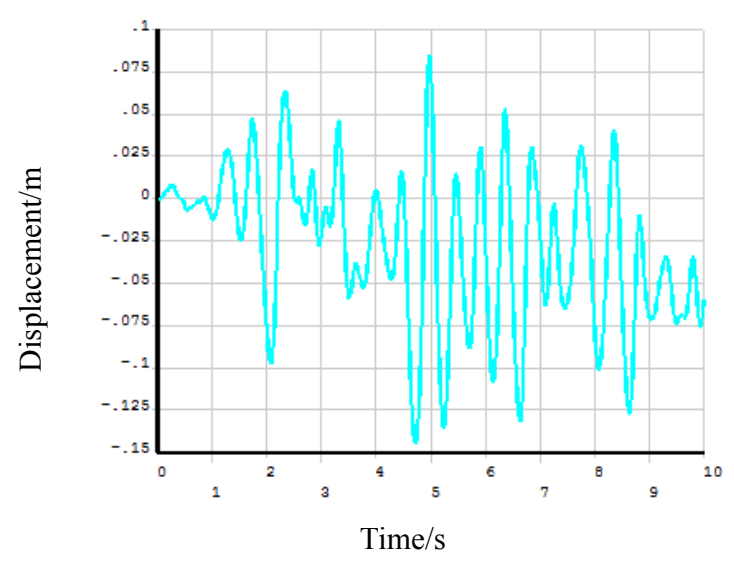

Fig. 7. Dynamic response of the maximum displacement of node 53

\section{EFFECT OF THE STIFFNESS OF THE SUBSTRUCTURE}

The above analysis clearly shows that the collapse acceleration decreases largely when the substructure is considered. To illustrate the effect of a different stiffness of the substructure to the collapse acceleration of the main reticulated shell structure, a further analysis of a different cross section of the substructure is carried out. In the numerical analysis, the tubular cross section of $\Phi 245 \times 10, \Phi 152 \times 6$ is used respectively for all the column of the substructure.

Fig. 8 shows the maximum dynamic displacement of the reticulated shell with substructure's cross section of $\Phi 245 \times 10$, $\Phi 152 \times 6$ and $\Phi 194 \times 8$ respectively. The figure shows that when the dynamic acceleration is less $4 \mathrm{~m} / \mathrm{s}^{2}$, the different stiffness of the substructure has little effect to the maximum displacement of the main reticulated shell. The maximum displacement of the main reticulated shell increases with the decrease of the stiffness of the substructure when the dynamic acceleration is larger than $4 \mathrm{~m} / \mathrm{s}^{2}$. Table I also clearly shows that the dynamic collapse acceleration of the main reticulated shell decreases with the weakened of the substructure. When the tubular cross section of $\Phi 245 \times 10, \Phi 194 \times 8$ and $\Phi 152 \times 6$ is used as the column of the substructure respectively, the dynamic collapse acceleration reduced $19.0 \%, 24.6 \%$ and $35.7 \%$ correspondingly comparing with the dynamic collapse acceleration of the main structure without considering the substructure. The maximum displacement is affected little by the stiffness of the substructure when the main reticulated shell collapses.

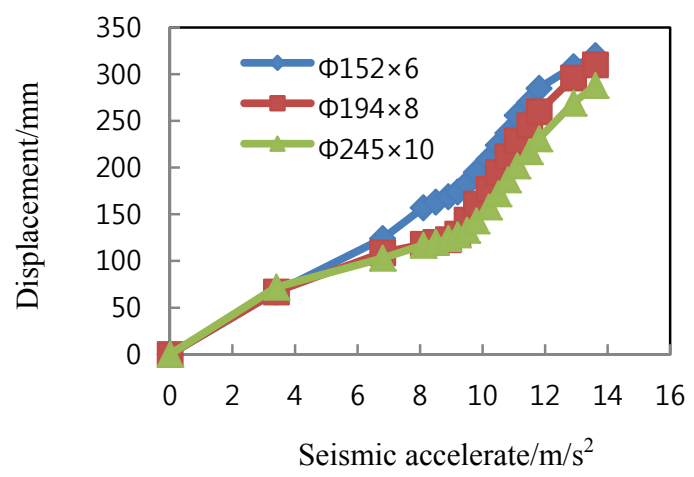

Fig. 8. Effect of the stiffness of substructure 
TABLE I. EFFECT OF STIFFNESS OF SUBSTRUCTURE.

\begin{tabular}{cccc}
\hline Section of column & $\Phi 245 \times 10$ & $\Phi 194 \times 8$ & $\Phi 152 \times 6$ \\
\hline $\begin{array}{c}\text { Dynamic collapse } \\
\text { acceleration }\left(\mathrm{m} / \mathrm{s}^{2}\right)\end{array}$ & 10.2 & 9.2 & 8.1 \\
Reduced ratio & $19.0 \%$ & $24.6 \%$ & $35.7 \%$ \\
$\begin{array}{c}\text { Maximum } \\
\text { displacement (mm) }\end{array}$ & 158 & 144 & 157 \\
\hline
\end{tabular}

\section{The Plasticity Members Distribution of the Main RETICULATED SHELl STRUCTURE}

With the increase of the dynamic acceleration, some members of the reticulated shell will reach into plasticity from elasticity, and this will affect the dynamic collapse acceleration of the structure. To demonstrate how the stiffness of the substructure affects the plasticity development of the member of the main structure, the investigation of the whole process of the plasticity development of members under increasing dynamic acceleration is presented by Fig. 9 and Fig. 10 .

Fig. 9 shows the ration of plasticity member of without considering the substructure and considering the substructure of different stiffness. The figure shows that for the same dynamic acceleration, the ratio of plasticity member of the reticulated shell with substructure is much higher than that of the reticulated shell without substructure and that the ration of plasticity member increases rapidly with the decrease of the stiffness of the substructure. When the dynamic acceleration is $3.4 \mathrm{~m} / \mathrm{s}^{2}, 1.5 \%$ of the members of the reticulated shell with a substructure of $\Phi 152 \times 6$ has reached into plasticity, but no plasticity members appear for the other conditions. When the dynamic acceleration reaches $5.1 \mathrm{~m} / \mathrm{s}^{2}$, the plasticity ratio of the member of the reticulated shell with a substructure of $\Phi 152 \times 6$ increases to $4.6 \%$, and the reticulated shell without substructure has no plasticity member still. Then with the increase of the dynamic acceleration, the plasticity members appear for reticulated shell of all conditions, and the plasticity ratio of members also increases. The plasticity ratio of members changes from $14 \%$ to $16.5 \%$ according to different support condition when the dynamic collapse of the main reticulated shell occurs. The investigation indicates that when more and more members reach into plasticity behavior, the stiffness of the main reticulated shell is reduced, and which finally causes the collapse of the structure. The plasticity members of the main reticulated shell with the weaker substructure appear much more early and the ratio of plasticity member increase much faster than that of the reticulated shell with stronger substructure and without substructure. Therefore, the dynamic collapse acceleration of the reticulated shell with weaker substructure is much less than that of the reticulated shell with stronger substructure and without substructure.

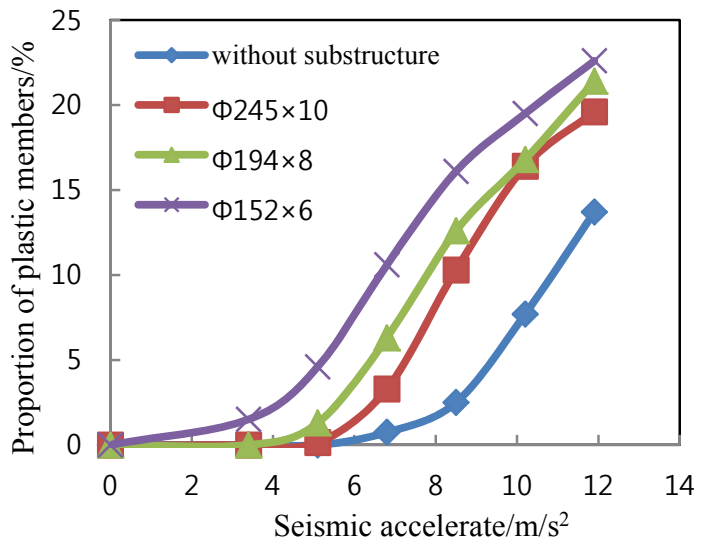

Fig. 9. The plastic ratio of the member of reticulated shell with and without substructure.

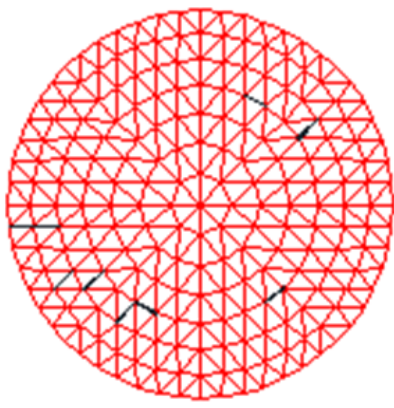

(a) $\mathrm{a}=5.1 \mathrm{~m} / \mathrm{s}^{2}$

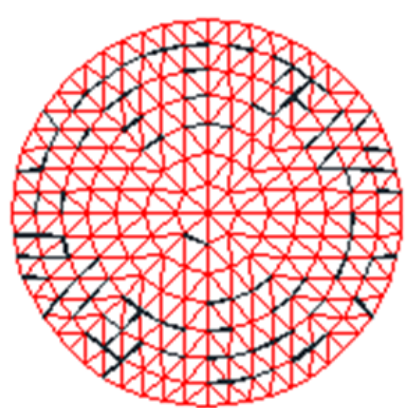

(c) $\mathrm{a}=8.5 \mathrm{~m} / \mathrm{s}^{2}$

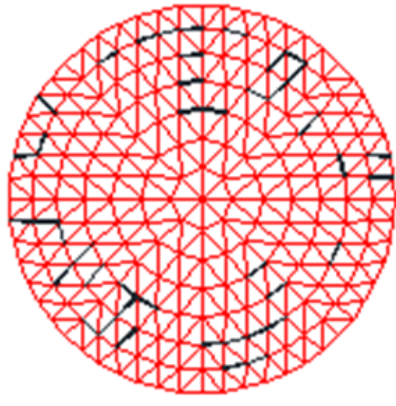

(b) $\mathrm{a}=6.8 \mathrm{~m} / \mathrm{s}^{2}$

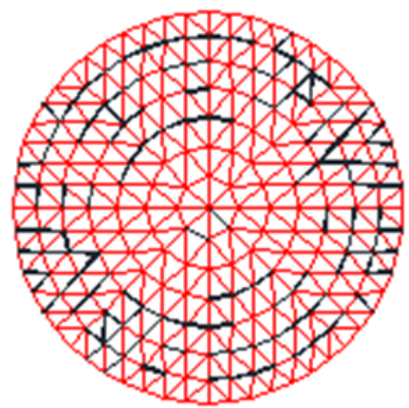

(d) $\mathrm{a}=10.2 \mathrm{~m} / \mathrm{s}^{2}$

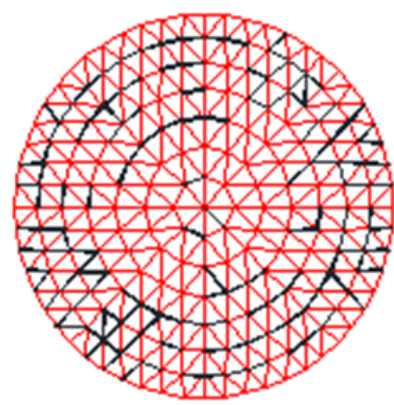

(e) $\mathrm{a}=11.9 \mathrm{~m} / \mathrm{s}^{2}$

Fig. 10. Development Process of the plasticity members of the reticulated shell 
Fig. 10 shows the development process of plasticity members of the main reticulated shell with a substructure of tubular cross section $\Phi 194 \times 8$, and it clearly demonstrates that with the increase of the dynamic acceleration, the more and more members of the reticulated shell reach into plasticity behavior from elasticity behavior.

\section{CONCLUSION}

This paper investigates the effect of substructure to the dynamic collapse of the reticulated shell. In the analysis, the geometric imperfections, the material and the geometric nonlinear of the structures are considered. The effects of the different stiffness of substructure to the collapse earthquake accelerations and the plastic member distribution of the reticulated shell are also investigated.

(1) The substructure will reduce the dynamic collapse accelerations of the main reticulated shell structure, and when the dynamic collapse of the reticulated shell structure is analyzed, the main structure and the substructure should be considered as an integral whole.

(2) The dynamic collapse acceleration reduced with the decrease of the stiffness of the substructure. This indicates that the stiffness of the substructure should have a certain stiffness to ensure that the main reticulated shell has enough earthquake resistance capability

(3) The plasticity members of the main reticulated shell with the weaker substructure appear much more early and the plasticity ratio of members also increase much faster than that of the reticulated shell with stronger substructure and without substructure. Therefore, the dynamic collapse acceleration of the reticulated shell with weaker substructure is much less than that of the reticulated shell with stronger substructure and without substructure.

\section{REFERENCES}

[1] S.Z. Shen. et al. Stability of Reticulated Shells. Science Press, Beijing, China, 1995.

[2] M. Fujimoto, and K. Imai, et al. Buckling Experiment of Single-layer Two-way Grid Cylinder Shell Roof under Centrally Concentrated Loading. Space Structures 5, Thomas Telford, London, 2002.

[3] W. Chen, G. Fu, and Y. He. Geometrically Nonlinear Stability Performance for Patial Double Layer Reticulated Steel Shell Structures. Space Structures 5, Thomas Telford, London, 2002.

[4] M. Zeinoddini, G.A.R. Parke, and P. Disney. "The Stability Study of an Innovative Steel Dome,” Int. J. Space Struct. vol. 19, no. 2, pp. 109-125, 2004.

[5] S. Jianheng. Stability of Braced Domes Under Dynamic Loads. Space Structures 4, Thomas Telford, London, 1993.

[6] S. Kato, T. Ueki, and Y. Mukaiyama, "Study of Dynamic Collapse of Single-layer Reticular Domes Subjected to Earthquake Motions and Estimation of Statically Equivalent Seismic Force", Int. J. Space Struct. vol. 12, no. 3/4, pp. 191-204, 1997.

[7] I. Ario, and T. Kaita, Dynamic Stability of Dome Structures with Homoclinic Orbit. Space Structures 5, Thomas Telford, London, 2002.

[8] F. Fan, S.Z. Shen, and G.A.R. Parke, "Study of the Dynamic Strength of Reticulated Domes under Severe Earthquake Loading”, Int. J. Space Struct. vol. 20, no. 4, 2005.

[9] A. Sadeghi. Horizontal Earthquake Loading and Linear/Nonlinear Seismic Behavior of Double Layer Barrel Vaults. International Journal of Space Structures, Vol. 19, No.1, pp. 235-244, 2004.

[10] T. Thkeuchi, and T. Orawa, et al. Response Evaluation of Medium Span Lattice Domes with Substructures Using Response Spectrum Analysis. Proceedings of the IASS, 2004.

[11] S. Jianheng, L. Hongmei, and A. Rahimi Noshnagh. Earthquake Effects on Single-layer Lattice Domes with Supporting Frames. Proceeding of IABSE-IASS2011, London, 2011.

[12] L. Liming, ANSYS Handbook for Finite Element Analysis. Tuinghua Publishing House, Bejing, 2005.

[13] F.P. Ulrich, "The Imperial Valley Earthquake of 1940”, Bull. Seismolog. Soc. Am. vol. 31, no. 2, pp. 13-31, 1941.

[14] B. Budiansky, and R.S. Roth, Axisymmetric dynamic buckling of clamped shallow spherical shells. Collected Papers on Instability of Shell Structures, NASATND1510, pp. 597-606, 1962. 